\title{
A Schnittmuster for Crafting Context-Sensitive Toolkits
}

\author{
Janis Lena Meissner ${ }^{1}$, Angelika Strohmayer ${ }^{1}$, Peter Wright ${ }^{1}$, Geraldine Fitzpatrick ${ }^{2}$ \\ ${ }^{1}$ Open Lab, Newcastle University \\ Newcastle upon Tyne, UK \\ $\{$ j.l.meissner2, a.strohmayer, p.c.wright\}@ncl.ac.uk \\ ${ }^{2}$ TU Wien (Vienna University of Technology) \\ Vienna, Austria \\ geraldine.fitzpatrick@tuwien.ac.at
}

\begin{abstract}
DIY-making can be an expensive pastime if makers are relying on ready-made toolkits, specialised materials and off-shelf components. Many prefabricated commercial kits seek to lower the learning barrier of making and to help beginners to successfully take their first steps in engineering. However, as soon as the novices become a little more advanced, these toolkits often do not fit the specific requirements of personal maker projects anymore. We introduce the idea of a Schnittmuster (or a meta-toolkit) as a novel approach to toolkit design that seeks to address these creativity-limiting factors as well as practical entrance hurdles. To demonstrate the adaptive power of the Schnittmuster concept, we discuss an exemplar in the context of capacitive touch sensing (FlexE-Touch). Implemented under the constraints of materials, user skill sets and making environments, we illustrate how the Schnittmuster facilitated four cheap and flexible toolkit instantiations for crafting custom touch sensor electrodes.
\end{abstract}

\section{Author Keywords}

Making; DIY; toolkits; empowerment; social justice.

\section{ACM Classification Keywords}

H.5.m. Information interfaces and presentation (e.g., HCI): Miscellaneous.

\section{INTRODUCTION}

A growing number of people are using digital maker technologies such as 3D printing, laser cutting and electronics hacking and the maker market is booming [14,39]. Make Magazine [40] and Maker Faires [41] have inspired many to embark on their own learning about how to make things. Over the years, many different tools and starter kits have become commercially available to serve the needs of this target group.

Most toolkits are designed to assist beginners in learning basic electronics concepts, physical computing and ubicomp-style engineering [11,19]. However, they all face

This work is licensed under a Creative Commons Attribution International 4.0 License.

CHI 2018, April 21-26, 2018, Montreal, QC, Canada

(C) 2018 Copyright is held by the owner/author(s).

ACM ISBN 978-1-4503-5620-6/18/04.

https://doi.org/10.1145/3173574.3173725 the challenge of dealing with a huge variety of users of different ages, backgrounds, and skillsets. Accordingly, the respective needs vary as well. Impairments [18], personal interest, and the amount of disposable time and money for hobbyist making all have an impact on gaining positive making experiences as well. Furthermore, every application context comes with its particular requirements. A pop-up making activity to engage children at a museum presents a very different setting to that of a brick-and-mortar maker space, a university lab facility or a traditional crafts studio.

The general accessibility of making and its toolkits thus becomes a far wider and more complex problem. Apart from learnability it also needs to address practical factors such as cost and availability of materials. Furthermore, a toolkit will only be used by individuals motivated to explore its functionality and creative capacities. One approach to make toolkits more attractive for not-yetmakers is an adaptable design that allows individuals to link new knowledge in making to their previous skills. In our research, we have employed DIY-making in highly varied contexts. To fit each project setting and the needs of those we were working with, we constantly needed to adapt the materials and tools of our toolkit. Thus, we have started to consider the toolkit concept on a functional level rather than in the form of a concrete, material implementation.

In this paper, we describe this novel flexible approach to DIY-making toolkits as a Schnittmuster (the German word for sewing pattern). Our intention is to use the word as a place-holding metaphor to provoke further discussion on what might be the most suitable term for a context-sensitive meta-toolkit. As such, the Schnittmuster concept makes a two-fold contribution by (i) providing a critique on the limitations of pre-fabricated toolkits, and (ii) offering an alternative design approach to developing custom toolkits. In the paper, we first examine literature and toolkit examples, to highlight common critiques and to motivate the need for, and define what we mean with, Schnittmuster. Then, we provide a case study of FlexE-Touch, which is an example of a Schnittmuster focused on capacitive touch sensing. The case study demonstrates four different toolkit instantiations which illustrate to the creative diversity of materials and settings. We will discuss how working in this way allowed us to avoid several limiting aspects of beginners' toolkits and to support playful adaptation and personalization of the toolkit components while embracing the craft aspects in interactive DIY-technology design. Within this case study, we provide a tangible description of 
the different elements that made up the Schnittmuster. However, our aim is not to present FlexE-Touch as a novel technology. Rather, our focus is on discussing the flexibility of this Schnittmuster concept and how this allowed us to adapt our materials and guidance to particular social contexts. This will be useful for people facilitating making activities for others, such as maker space managers, workshop organisers or designers working in collaborative settings.

\section{BACKGROUND AND RELATED WORK}

In this section, we contextualise our critical account within the existing work on toolkits and how HCI researchers have been addressing their limitations. First, we provide a brief history of toolkits looking at the dynamic interconnections between the educational and commercial sector. Second, we address how toolkits have been critiqued for being limiting in use and creativity. Finally, we review the ways in which HCI has started to move beyond toolkits through the design of the kit-of-no-parts and the untoolkit.

\section{History of Toolkits}

Toolkits have a long tradition, both in teaching electronics and in their commercialisation as products. In Germany for instance, so-called 'experiment kits' became popular as early as the year 1900 [3]. Examples for such home laboratories designed to fit into a box are the KOSMOS Baukasten Elektro (1921, cf. figure 8 on page [3:404]) or the Braun Lectron (1967, [38:611]). These kits usually comprised a set of educational materials for self-paced study. Instructions guide the amateur learner (usually children) in how to use a provided selection of materials to conduct experiments and create circuitry.

The technological advances in the late $20^{\text {th }}$ century made it possible to integrate sensors, actuators and even computing resources into these kit-type products. These possibilities coincided with a growing interest within computing science in ubicomp and tangible user interfaces [28]. Pioneer toolkits such as Phidgets [11] or iStuff [1] were introduced to train students for the new challenges of designing and implementing digital technologies beyond desktop applications. The development of Phidgets [11] for example was motivated by the lack of electronics and engineering know-how among computer scientists. According to Dumas, these toolkits were "typical examples of the hardware prototyping approach, offering developers a set of hardware components, such as buttons, knobs or LEDs, and software drivers to use those components" [8:49].

These first successful trials with computing students in the academic context were soon echoed by the commercial market. The emergence of the Maker Movement provided a new market sector for start-ups to create and sell new tools and starter kits for makers and those who want to become makers. Brands such as Arduino [42], SparkFun [43], Adafruit [44] and micro:bit [45] have been particularly active in creating toolkits for electronics projects. Examples of this product range include highly specialised clip-and- play boards (such as the Makey Makey [46] for translating touch sensor inputs into keyboard commands, or littleBits [47] using magnetic and colour-coded building blocks to create circuitry) as well as more open platforms (eg. microcontrollers such as the Arduino Uno [48], LilyPad Arduino [49], or Teensy [50]) which are often sold together with a range of standard off-the-shelf electronics components.

With this growing palette of toolkits available, HCI researchers have been very active in exploring their capacities within different social contexts. The Makey Makey for example was used by Rogers et al. [29] to involve older adults as creative co-designers in design workshops, and by Somanath et al. [34] to engage 'at-risk' high school students. There has also been a significant amount of work creating specialised toolkits with a specific focus on certain groups of people (eg. [21] working with occupational therapists), technologies (eg. [5] creating DIY ambient displays) or artefact creation method (eg. [30] combining tangible prototyping with Lego).

However, little work has discussed how the design of specialised toolkits can include or exclude certain groups of users based on its materiality. Considering the traditional interconnections between commercial and academic toolkits, it seems surprising that there has been a lack of attention to very practical factors such as cost, access to materials and required amounts of disposable time. As we discuss in the next section, most critiques have centred on balancing the tension between facilitated learning and allowing space for personal creativity.

\section{Critiquing Toolkits}

The basic idea of toolkits is to simplify certain parts of the engineering and programming processes. Apart from this kind of abstraction being an established practice in software engineering, there are usually two main motivations for this blackbox-type approach $[1,8,11]$ : (i) modules can be reused and help to save time, and (ii) modules can be used without knowing every technical detail enabling beginners to use them. While assisting novices is a noble aim, it also comes with massive challenges. With the design objective to simplify complex processes by pre-defining functional modules (or effectively blocks for a limited number of functionalities), the toolkit designer needs to envision what the user will or can do with it. However, it is hardly possible to anticipate all possible use scenarios, and thus, the support which toolkits provide necessarily comes at the cost of introducing constraints.

These limits in use appear on a socio-technical level. On a technical level, some HCI work criticised the level of abstraction (as in terms of configuration, set-up and API) for being too static to fit the needs of specific design tasks (eg. [8,11]). Others, more concerned with the experiences of toolkit users, state that the dependency on a modular system constrains what they build and how they think [25]. Furthermore, toolkits are used in varied social settings and thus need to deal with a huge variety of users of different 
ages, backgrounds, and skillsets. Accordingly, the respective needs vary as well. For example, an adult might be more intimidated by making mistakes than a child who is just playing around. However, a 6-year-old has a different attention span and motor abilities than a 60-year-old.

While previous research has explored how toolkits can be powerful means to facilitate access to science, technology, engineering, and maths (STEM) knowledge in schools and universities (eg. [11,12,34]), issues get more complex and political outside formal educational institutions. Social divisions in society and the marginalisation of different communities based on their abilities, gender, age, race, or social class also tend to reflect in the demographics of those participating in the Maker Movement. Many of those engaging in makerspaces are socially more privileged individuals; mostly male, middle-class, white and welleducated [2,6,37,39] and do not represent the communities which traditionally have been addressed by research through social justice agendas [7,24]. If toolkits are supposed to serve as entry points for not-yet-makers into the world of making, their design, material implementation and the ways how they constrain become political as well.

Can a single toolkit address this real-world complexity? It is hard to find the right balance of support and creative freedom. Perner-Wilson [25] and Mellis [19,20] addressed this issue and respectively explored alternative approaches: (i) the kit-of-no-parts; (ii) the untoolkit.

\section{The kit-of-no-parts}

Perner-Wilson proposed the kit-of-no-parts [25], where offthe-shelf electronics are first unpicked in their electrical properties and then recreated from crafts materials. Working with e-textiles, the kit-of-no-parts embraces the craft side of DIY electronics construction activities. The focus here is on recreating different existing off-the-shelf components and thus on individual material exploration. The kit-of-no-parts approach can be described as a specific form of inquiry through the use of a chosen set of tools and materials [26,27]. It allows for a huge space of creative DIY electronics implementations, but this freedom relies on people's dedication to fully unpicking and understanding what might seem like a mystery to them at first. People without a technical background tend to be quite intimidated by DIY technology [12,37]. Perner-Wilson tries to overcome this hurdle by differentiating between crafting and engineering in terms of the materials. This can indeed be a productive tactic for attracting people's interest in a topic that they might have previously ignored [4], but we believe that there needs to be some more guided first steps before novices can embark on the mission to create anything from anything.

\section{The untoolkit}

Another alternative approach to toolkits is the untoolkit introduced by Mellis [19]. This is described as a set of hardware and software tools that provide 'an accessible toolchain' for, for example, the programming of microcontrollers. The untoolkit works in operational steps, therefore partly turning its modules into tools (or redefining them as materials, as was done with microcontrollers in this paper). Mellis' main argument for the untoolkit is that it 'loads the expense primarily onto the tools themselves' [19:88]. In the context of microcontrollers, he did so by offering an easy USB interface to program very cheap ATtiny microcontrollers. This would then allow novice makers to use cheaper and smaller standard electronics components together with craft materials instead of premanufactured bulky toolkit modules. We understand the untoolkit to be a great way of minimising the costs of making with off-the-shelf electronics components, while simultaneously reducing the dependency of novice makers on commercial brands and their products. However, the novice still remains dependent on some readymade electronics and someone who pre-selects these for them.

\section{Summary}

Reviewing the history of toolkits (with a focus on those developed for educational purposes) highlights the dynamic interplay between the commercial and academic worlds that fueled the design and distribution of such products. Indeed, previous work has illustrated how kit-based approaches can be a powerful means to teach electronics through creative and self-paced experimentation with the provided toolkit blocks. However, most of this work was conducted in settings formally framed around learning (eg. in schools or universities) or optimized by the researchers to serve the study of learnability with or creative adoption of toolkits. Practical factors constraining the use outside these ideal conditions for learning have often been ignored. Most critical approaches to toolkits have addressed the technical problem of hiding complexity at the cost of constraining creative use on the level of an individual toolkit user. Yet, looking at less-advantaged settings or where toolkits are not yet used raises more political questions around equal opportunities to access education resources. We argue that the constraints appear both on a technical/material and on a social/cultural embedding of these practices.

The kit-of-no-parts has contributed a powerful way to explore electronics through re-crafting. It makes learners materially independent but might not provide enough guidance for complete novices (material freedom at the cost of little guidance). The untoolkit, in contrast, continues to use cheap ready-made standard components and contributes new tools to make these elements more accessible for novices. While craft can be used around these elements, it still frames the learning in the tradition of engineering (material constraints and a specific modality of guidance).

In response to the raised issues and gaps in the existing literature, we developed an alternative approach which is motivated by three factors: (i) facilitating learning through providing enough guidance for complete beginners to learn about an electronic concept, (ii) material freedom by letting toolkit users choose and replace materials or tools, and (iii) 
flexibility by taking practical factors into consideration that can make a difference for those who might otherwise not use electronics toolkits. We call this approach a Schnittmuster and will now provide a definition of our framework that incorporates elements of the history and critiques of toolkits, while simultaneously going further to incorporate valuable social elements into the discussion.

\section{DEFINING SCHNITTMUSTER}

Schnittmuster is the German word for sewing pattern, but literally translates to 'cut pattern'. Schnittmuster thus focuses on selecting and preparing the needed components rather than the process of their later composition. The word 'sewing pattern' already defines that the cut-outs will be used for sewing. 'Schnittmuster', in contrast, only defines that components are cut out and keeps it open if these will be assembled later, leaving room for a variety of techniques (eg. sewing, gluing, or fusing). Therefore, despite also being mostly used for textile purposes in German, Schnittmuster is still a relatively neutral term. It not only applies to textiles, and is, for example, also used in the metal-processing industry. However, opposed to the general English term pattern (which could also refer to visual or geometric motifs such as spirals, waves and tilings), the German Schnittmuster is unambiguous in that it is functional rather than decorative.

Based on these characteristics, Schnittmuster highlights material flexibility while maintaining a focus on selecting appropriate functional components. In other word, it allows creative interpretation and adaptation to particular contexts and frames the aspects to think about and their relation to each other. Just as a sewing pattern can adapt its sizes for creating clothing for a very petite or large person, a Schnittmuster for toolkits can modify the materials of its components to be used by knitters, quilters, glass artists, hobby tinkerers, and others. For doing so, we consider the toolkit concept more on a functional level than in the form of a concrete material implementation. We argue that a Schnittmuster is not necessarily a toolkit in itself. Rather, it is a method to create custom toolkits for the same technology in different application contexts. As such Schnittmuster is a context-sensitive meta-toolkit, that allows us to choose materials that fit the project setting as well as the toolkit users' skills and knowledge level.

\section{CASE STUDY: THE FLEXE-TOUCH SCHNITTMUSTER}

To exemplify how the concept of a Schnittmuster can come into action, we present a case study under the name of FlexE-Touch. This particular Schnittmuster is about building capacitive touch sensor electrodes from different materials and facilitating this in adaptive ways to match project settings and the users' skills and knowledge levels.

\section{Practical Background to this Work}

Our work on FlexE-Touch initially emerged from the very practical constraints of a design project [15]. The aim was to integrate touch sensor electrodes into hand-crafted textile rectangles. Simultaneously, it was important that the sensor integration would not interfere with the crafting process of the textile designers. It was important that the final artefact could be installed flat or wrapped around building pillars or trees, that the users of the object could freely move while interacting with it through touch, and that the materials used for the electrode design were as cheap as possible. To meet all these constraints, low-cost capacitive touch sensors were created out of aluminum foil which was ironed onto the back of the finished textile rectangles and connected to an Arduino Uno (which was already owned by the maker) via wires soldered to paper clips. In this way, the sensors were attached one after the other. The final design artefact was cheap to produce as no conductive yarns or fabrics were used, and it remained sufficiently flexible for different installation contexts.

Since this project, the method has evolved and been refined over the course of different research projects, public engagement activities and personal craft explorations. As we will point out later in this paper, the application context of each of these has been very different. In some instances, we could use FlexE-Touch to facilitate pop-up making with novices who happened to drop by at the right place at the right time. In others, we could collaborate with crafts experts directly in their workshops and expert domains. The idea of Schnittmuster came out of reflecting on these various cases. Due to the changing project settings and the different groups of people we worked with, we constantly needed to adapt the toolkit in terms of materials and how we employed it. At first glance, the differently looking project outcomes might not seem to have much in common. Yet, they all are instances of FlexE-Touch. They were built based on the same set of functional components and by interpreting which materials and fabrication techniques might be most appropriate for the particular setting.

\section{FlexE-Touch}

Capacitive touch or proximity sensors exploit the physical characteristic of the human body being electricallyconductive and thus having the ability to interfere with electrical fields. A capacitor is an electrical component that classically consists of two terminals. For example, it could be two metal plates in a parallel position to each other that are separated by a non-conductive or dielectric medium (for instance air). Connected to a power source an electric field develops between the plates. Capacitive touch sensors register changes in this electrical field and instead of the two metal plates they use an electrode that builds an electric field with a human hand, for example.

In HCI research, different specific uses of capacitive sensing for prototyping have been documented (eg. $[10,13,22,23,32,33])$. FlexE-Touch however functions as a Schnittmuster for tailoring different ways to build sensor electrodes for capacitive touch sensing. Thus, we need to define its functional components and which characteristics they have for the engineering process. Applying this highlevel view, FlexE-Touch consists of up to five basic toolkit 
components for creating a system of a touch sensor electrode connected to a signal-processing unit (see figure 1). In this paper, we will only focus on the sensor elements, as we want to illustrate the multifaceted creative capacity within this set of simple components. Due to this, we do not go into the details of the variety of possibilities for different actuators.

FlexE-Touch is explicitly not restrictive or exhaustive in terms of materials or tools that can be used for each of the functional components. In this pick-and-mix style, custom toolkits can be realised through assembling materials that make sense in the specific setting. Apart from needing to fulfil some functional criteria for each of the basic components, there are no limits set to what the used materials could be: They can be hard or soft. They can comprise off-the-shelf artefacts or materials that require manipulation through crafting. They can adapt to working with wool, wood, fabrics, glass, ceramics or metal.

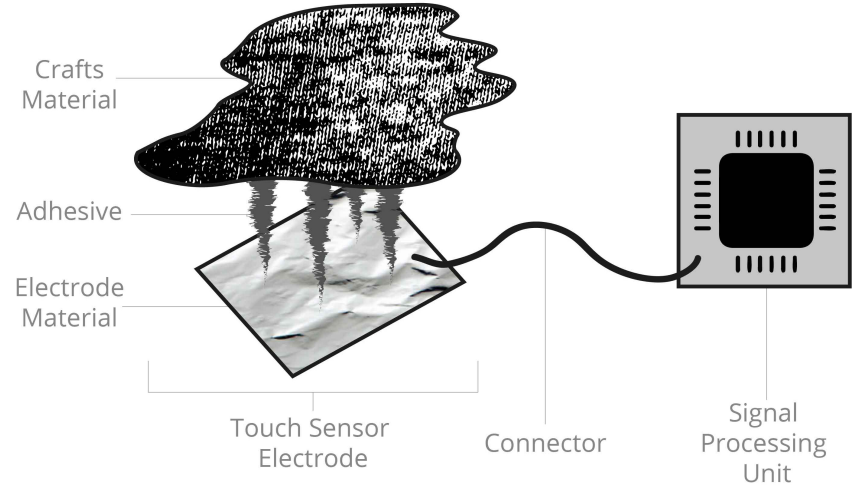

Figure 1. General FlexE-Touch scheme providing an overview of all functional toolkit components.

\section{The Schnittmuster}

Since we are using the German word Schnittmuster to reflect on the materialities of our capacitive touch sensors, we want to provide an explanation and description of the literal Schnittmuster for FlexE-Touch. FlexE-Touch consists of the following basic components: (i) a working craft material, (ii) an adhesive, (iii) an electrode material, (iv) a connector, and (v) a signal processing unit (see figure 1). In this section, we describe each of these components and expand on their functional requirements in detail. We also provide some examples for possible material choices. However, again, this is not meant to be an exhaustive list.

\section{Craft Materials}

A key aspect of FlexE-Touch is that it technologically augments craft outcomes and turns them into sensors. This means that in the original method the touch sensor electrode is subsequently attached to a craft artefact rather than disrupting the process leading to it. The initial craft activity does not need to be modified in any way to integrate the electrode material. Thus, the maker can use any craft materials and techniques to produce an artefact that could be turned into a capacitive touch sensor. Examples of potential materials include but are not limited to: paper and cardboard for drawing or creating collages, fabric and threads for sewing or embroidery, yarn for knitting or crochet, and wood for carving or engraving.

Most craft materials are not conductive and will therefore serve as the dielectric medium in the capacitor design. In our experience, relatively flat craft designs work very well for proximity sensing. However, the exact maximum distance between hand and electrode will depend on the size and sensitivity of the sensor. If an electrically conducting craft material is used (such as metal or conductive ink), the crafts material simultaneously serves as the electrode material and no adhesive is needed.

\section{Electrode Material}

The sensing part of the electrode needs to consist of a conductive material. Ready-made conductive ink or fabric are good but often costly options, being very specialised products. In most cases, there are cheap alternatives available that sufficiently serve the purposes of an electrode. Depending on what the maker has at hand, the electrodes could be cut-outs of aluminum foil, drawn shapes coloured in with a soft lead pencil or helically bent copper wires. The shape design of the electrode can be also flexible. In our projects, we mostly worked with areas of electrode material in a comparable size to a human hand. However, it could also be a pattern of interconnected lines. If a straight wire is used, this might reduce the reach of the sensor, so that only a finger in closer proximity is detected.

\section{Adhesive}

Any suitable adhesive medium can be used to attach the electrode material onto the working crafts material. By suitable we refer to the physical properties of the used materials as well as to the project objectives. A glue stick might be the easiest way to stick foil onto cardboard, but it can leave grease stains on fabrics and is less reliable than using an iron-on adhesive or sewing electrodes into place. Again, an adhesive is only needed if the craft material is not conductive and the electrode material is not self-sticking.

\section{Connector}

Depending on the materials at hand, there are also different possibilities of how the electrode can be connected with the processing unit. Just as the adhesive must fit with the craft material, the connector must be suitable to the electrode material. On the one end, there needs to be a good connection with the electrode material. Soldering a wire to aluminum foil only works with the right types of flux and solder. A novice thus might find it easier to use a crocodile clip. However, the crocodile clip might be too bulky for flat projects and could be replaced by a paper clip (which can be easily soldered to a wire). On the other end, the connector also needs to fit the input pins of the processing unit. While Arduino Uno is made for connecting components with jumper wires, LilyPad Arduino can create connections by threading, tying or sewing conductive thread through its port-holes. 


\begin{tabular}{|c|c|c|c|c|c|}
\hline Use Cases & Crafts Material & Adhesive & $\begin{array}{l}\text { Electrode } \\
\text { Material }\end{array}$ & Connector & Processing Unit \\
\hline $\begin{array}{l}\text { Tools for } \\
\text { Wools }\end{array}$ & $\begin{array}{l}\text { Yarn: } \\
\text { Knitting, crochet, and } \\
\text { machine knitting with } \\
\text { different types of yarn }\end{array}$ & Fusible web & Aluminum foil & $\begin{array}{l}\text { Wires soldered } \\
\text { to paper clips }\end{array}$ & $\begin{array}{l}\text { Arduino Uno and } \\
\text { MPR121 } \\
\text { breakout boards }\end{array}$ \\
\hline $\begin{array}{l}\text { Partnership } \\
\text { Quilt }\end{array}$ & $\begin{array}{l}\text { Fabric: } \\
\text { Quilting with different } \\
\text { types of fabrics, scrap } \\
\text { fabric, paper templates, } \\
\text { padding, embroidery } \\
\text { floss, and buttons }\end{array}$ & $\begin{array}{l}\text { Fusible web, } \\
\text { machine sewing }\end{array}$ & $\begin{array}{l}\text { Aluminum foil } \\
\text { strengthened with } \\
\text { iron-on cotton }\end{array}$ & $\begin{array}{l}\text { Wires soldered } \\
\text { to paper clips }\end{array}$ & $\begin{array}{c}\text { Bare Conductive } \\
\text { TouchBoard }\end{array}$ \\
\hline Light Writer & $\begin{array}{l}\text { Glass: } \\
\text { Layers of CNC-cut } \\
\text { glass fused in a kiln }\end{array}$ & $\begin{array}{l}\text { None } \\
\text { (due to glass } \\
\text { fusing) }\end{array}$ & Bent copper wire & $\begin{array}{l}\text { Copper wires } \\
\text { and crocodile } \\
\text { clips }\end{array}$ & $\begin{array}{l}\text { Bare Conductive } \\
\text { TouchBoard } \\
\text { with an } \\
\text { Arduino Shield }\end{array}$ \\
\hline $\begin{array}{l}\text { Pop-Up } \\
\text { Making }\end{array}$ & $\begin{array}{c}\text { Mixed Media: } \\
\text { Laser-cut baseplates } \\
\text { (card board, wood, } \\
\text { acrylics); decorated } \\
\text { with crafting materials } \\
\text { such as paper, card, } \\
\text { pens, yarn, embroidery } \\
\text { floss, and stickers }\end{array}$ & $\begin{array}{l}\text { Different glues and } \\
\text { tapes, needles and } \\
\text { thread for sewing }\end{array}$ & $\begin{array}{l}\text { Aluminum foil } \\
\text { copper tape }\end{array}$ & Crocodile clips & $\begin{array}{c}\text { Bare Conductive } \\
\text { TouchBoard }\end{array}$ \\
\hline
\end{tabular}

Table 1. Material interpretations of the FlexE-Touch Schnittmuster and its components per use case

\section{Processing Unit}

The processing unit can be any component that is able to register capacitive touch sensing signals and transform this into a desired output (such as sound or light). While most FlexE-Touch components work with non-technological resources, the processing unit is the most specialised part of the kit. However, it does not necessarily need to be the most difficult one for novice makers. Some controller board products such as the Bare Conductive TouchBoard [51] work out-of-the-box even if the crafted electrodes are only temporarily clipped to it. By default, it translates capacitive touch input into sound output and in our experiences novices feel quite comfortable with this default set-up. Again, specialised products are often more expensive than more open ones that require programming; examples of these include controller boards such as Teensy [50] and Arduino [48] using the CapSense library [52] in their code or breakout boards such as MPR121 [53] in their hardware. However, as the maker becomes more experienced, these general boards will also offer more design possibilities.

\section{INSTANTIATING THE FLEXE-TOUCH SCHNITTMUSTER}

After introducing FlexE-Touch as a Schnittmuster for building DIY capacitive touch sensor electrodes, we present four different instantiations: (i) a design exploration into urban knitting, (ii) a collaboration with a charity to create a living archive, (iii) a project for personal craft practice development, and (iv) an example of how FlexE-Touch can be used as a tool for public engagement.
Here we focus on the social elements and the contextual surroundings of the projects the Schnittmuster was implemented in, and also provide brief descriptions of the materialities of the ways in which we used FlexE-Touch. Based on the social and contextual constraints, as well as skill levels and interests of participants, each of these usecases constitute different material interpretations of the functional components (see Table 1 for an overview).

\section{Tools for Wools}

'Tools for Wools' was the title of the previously mentioned project from which FlexE-Touch originates. It involved an explorative study of urban knitting ${ }^{1}[16,17]$ and aimed to identify design opportunities for embedding digital technology in this craft practice. Eventually, this led to the development of a touch-interactive collaborative urban knitting installation (see Figure 2 for the finished prototype and interactions surrounding it). A call for participation on Twitter recruited 26 urban knitters from 7 different countries. Each of them created and posted a hand-crafted rectangle. They also provided a photo of themselves with their contribution and a text with background information. All rectangles were assembled to create a flexible blanket $(175 \mathrm{~cm} \times 120 \mathrm{~cm}$ in size) and touch sensors were attached to the backside of each crafted panel. In this instance,

\footnotetext{
${ }^{1}$ Urban knitting is also known as yarnbombing or guerrilla knitting. Yarn and traditional techniques such as knitting, crochet, or embroidery are used to modify urban infrastructure. For example, trees are covered in knitting.
} 
aluminium foil was used as the electrode material, iron-on fusible web as the adhesive, wires soldered to paper clips as connectors and MPR121 breakout boards together with an Arduino Uno as the processing unit. The microcontroller was programmed to translate the touch input into a Bluetooth signal that would prompt an Android app to display corresponding information. The installation let the audience explore the artefact by touching its craft details and comparing it to the provided background information.

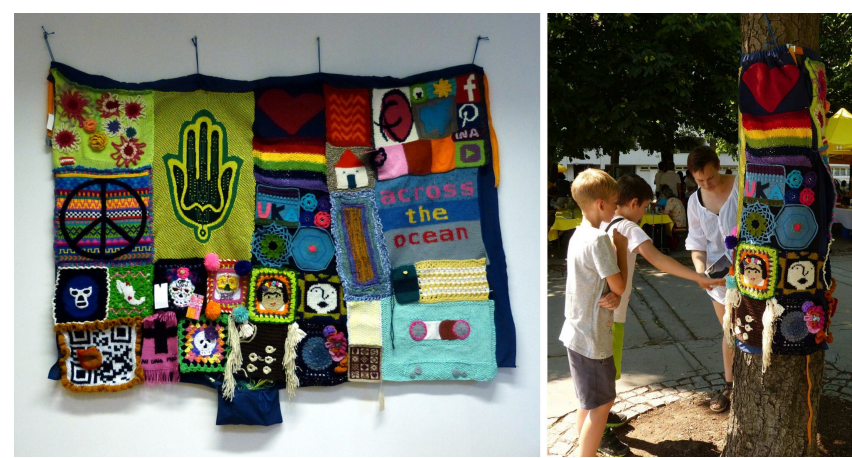

Figure 2. The Tools for Wools prototype

This project was constrained in terms of funding as well as the need to correspond with existing practices and formats of real-world urban knitting installations. Furthermore, the collaboration with the urban knitters needed to take place distributed over the globe. While the prototype ended up quite hacked in nature, FlexE-Touch managed to bring all these factors together. The researcher/designer/maker was a novice to electronics and programming microcontrollers at the start of the project, but managed to learn the skills motivated by working within a crafts context she felt passionate about. Here, she was both the electronics learner and a facilitator by distributing tasks and outsourcing the crafts material processing to external project contributors.

\section{The Partnership Quilt}

Another textile application of FlexE-Touch was a research project that lead to the creation of the Partnership Quilt. We had the opportunity to collaborate with professional quilters and a charitable sex work support service. The project emerged from the charity staff's efforts to employ handicrafts as a calming and mindful activity. Many of their service users would go through difficult times, and as described by [36], sitting down together at drop-in sessions for some quilting over a cup of tea worked well to create a relaxed atmosphere and space for collective creativity as much as for individual mindful practice. As the quilting blocks piled up, two professional quilters got involved to help the group of charity staff and service users to assemble the blocks to turn into a thicker padded blanket. During this process, we introduced the idea of using FlexE-touch to turn the quilt into an interactive piece of craftwork. Together in this highly diverse group that happened to form around a craft activity, we then created a 'living archive of sex worker voices' [36]. The resulting prototype looked just like a quilted blanket. However, touching one of the quilted rosettes triggered an audio clip that would allow the person interacting with the quilt to learn about its story, and the many layers of stories of those involved in the project.

For this, we had recorded a reflexive group conversation and saved audio-snippets on the micro-SD card of a Bare Conductive TouchBoard (signal processing unit). The other toolkit materials were very similar to the ones used in the Tools for Wools project. Again, the touch sensors were made of aluminium foil strengthened by iron-on cotton (electrode material) and attached to a layer of padding on the backside of the quilted blanket. However, building on the professional crafts expertise of our partners, we could improve the toolkit for quilting: To position the electrodes aligned with the blocks, they were first ironed onto the fabric and then fastened by machine sewing. Thus, FlexETouch itself became a form of quilting ${ }^{2}$. The connections consisted of wires soldered to paper clips (stitched down to the aluminium foil) on the electrode side and to the Touch Board electrode pins on the processing side.

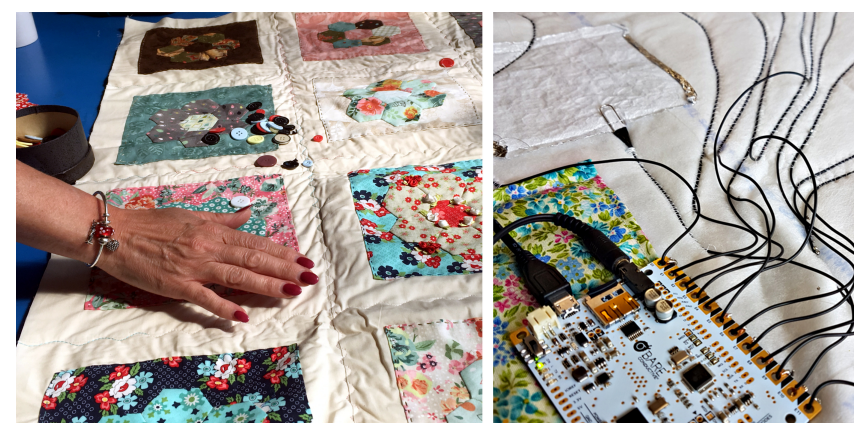

Figure 3. The Partnership Quilt

This Partnership Quilt project faced constraints in funding and in time as it was difficult to bring together everyone at the same time. Furthermore, the skill sets of those involved were highly varied, some being novice crafters, some working as professional pattern designers and some being more experienced with digital technologies than with sewing. This adaptation of FlexE-Touch for quilting was only possible due to active engagement and mutual learning on all sides. We researchers were the facilitators of the digital making part of this project, yet we also were learners. We learnt just as much from the charity staff, the service users, and the quilters about the craft and processing the toolkit materials in sturdier ways, as they learnt from us about capacitive touch sensing and operating the controller board. Using mostly traditional textile crafting materials and quilting techniques (eg. embroidery, applique, or machine sewing) under the guidance of the professionals, lowered the participation barrier significantly and afforded curiosity on all sides based in how the unusual assemblage of materials would result in an interactive artefact. Furthermore, using a specialised controller board product (which was provided by the researchers) where little

\footnotetext{
${ }^{2}$ Quilting is the craft of creating a padded fabric of multiple layers
} 
modification other than exchanging the MP3 files was necessary, helped reduce feelings of intimidation and create interest in how the controller itself, but also the quilt, work.

\section{Light Writer}

We also used FlexE-Touch in more explorative settings with completely different materials. In collaboration with crafts researchers, artists and a local glass museum, we had the opportunity to use the specialised workshop facilities for experimenting with how to make glass conductive and thereby useable for touch interaction. The first author participated in a workshop series that brought together people with diverse backgrounds, skills, interests and project aspirations. As the organising crafts researcher retrospectively wrote in a report [31]: "The activities, though not explicitly framed as collaborative, would then act as inter-institutional knowledge exchange opportunities. With backgrounds in digital craft, fashion, glass, computer science, $3 D$ product design, fine art and creative technology, diversity was certainly achieved and the sharing of knowledge and approaches across these domains was evident in the conversations, demonstrations and facilitations that I witnessed in [the] workshop sessions. This opportunity for participants to begin to understand practices and processes first hand in which they had had no previous experience was universally appreciated and extended both the aspirations and the practice of many."

Given this dynamic interplay and mix of expertise, the participating author kept constantly oscillating between roles of a learner and a facilitator. In her case, she shared her knowledge on designing touch sensor electrodes and using the Bare Conductive TouchBoard. However, she could also extend her knowledge on glass processing by learning from the organisers and other participants. She challenged herself by changing the default actuator output from sound to light and connecting an RGB-colour LEDstrip. She felt this to be a more fitting mode of interaction for glass as a translucid material. Eventually, this exploration resulted in an artefact which was titled the Light Writer (see figure 4), a semi-transparent fused glass object resembling a piano with three static keys in the colours red, green, and blue. The keys would serve as RGB component inputs for mixing and "writing" coloured light.

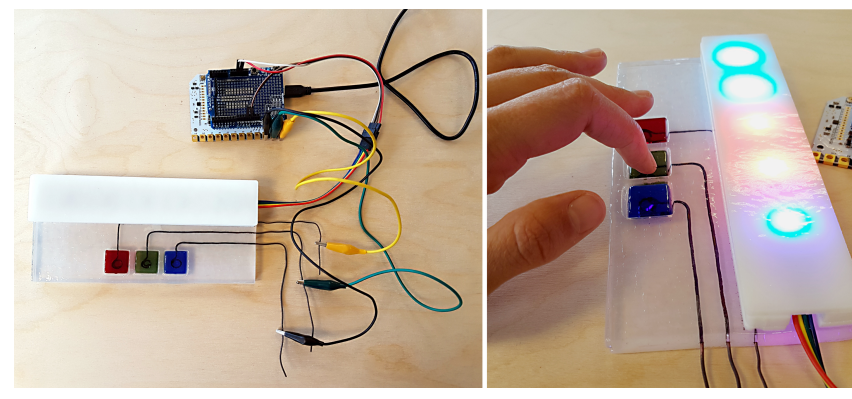

Figure 4. The Light Writer

Again, the light writer follows the design principles of FlexE-Touch. However, the materials used for the FlexE-
Touch toolkit components were very different. Here, glass was the working crafts material, the keys were made of smaller pieces of red, green and blue coloured glass, fused on top of the electrodes. For the electrode material, the Light Writer used thick copper wire whose ends were circularly bent to create a larger electrode area. The other ends of the copper wire stick out of the glass object to allow clipping crocodile clips to it (forming the connectors).

\section{Public Pop-Up Making}

While the Light Writer was developed in a rather exclusive creative think-tank with abundant resources, we also present a contrasting case where FlexE-Touch was used out and about for engaging complete novices of varying ages, interests, skills, and abilities. Based on its pick-and-mix approach, we designed a pop-up making activity for public outreach that embraced individual creativity and did not require any previous knowledge. Learners were assisted to create a personal touch sensor electrode and connect it to a TouchBoard to trigger the sound of an animal. A facilitator provided basic guidance by demonstrating some examples and outlining the steps needed to design, build and test an electrode. We ran the activity in four different settings: (i) a stall at a festival in a local park, (ii) a guest activity at a girl guide unit meet-up, (iii) a re-design workshop for novice makers with disabilities, and (iv) an activity at the summer party of a community arts organisation.

To get participants started quickly, a selection of 'base plates' in different materials and colours were laser-cut in preparation for the engagement. A participant then chose a base plate and decorated it using the crafts materials and tools provided by the facilitator (eg. paper, card board, yarn, pens, and stickers). All available materials were placed visibly on the table. In this way, the participants could personalise the activity to their personal preferences in materials and crafting techniques as well as to their skills and abilities. Indeed, while we used just two different cutting templates for producing the base plates, each created touch electrode was truly unique (see figure 5).
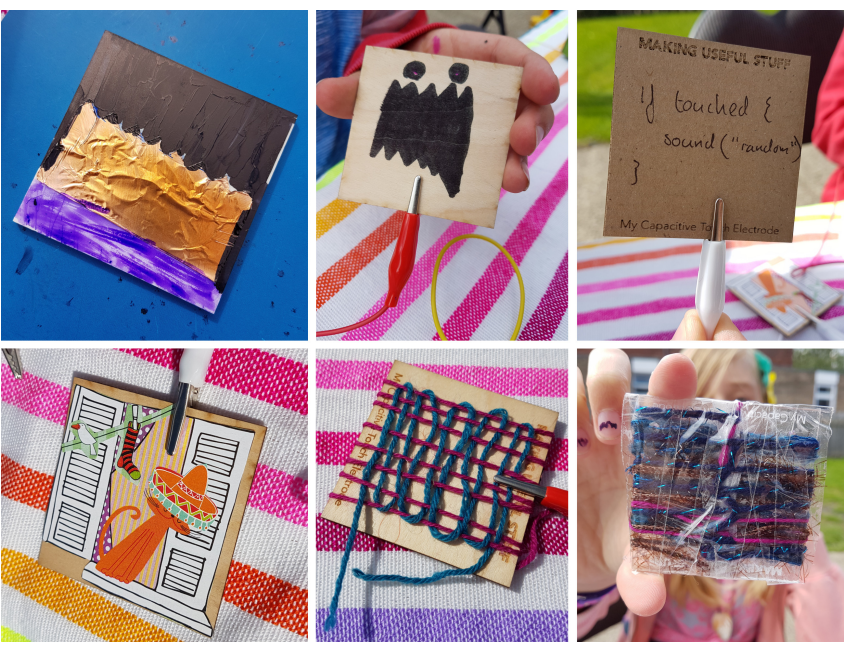

Figure 5. Examples of touch sensor electrodes creations 
While we provided a large variety of materials to choose from, the resources were practically limited by whatever could be put and transported in boxes. Another constraint in this setting was time. The DIY activity needed to be brief as participants could not be expected to spend more than 10 minutes on it. Using FlexE-Touch helped to meet all these factors. While some of the components were pre-decided by the activity facilitators (such as using the TouchBoard for the signal processing and crocodile clips as connectors), it was left to the participants to decide on the decorative crafts, the electrode material, and the adhesive they wanted to use. They could use the prepared base plates or try to recreate provided sample designs, however, they did not need to and were encouraged to use materials just as they liked. Providing such a flexible toolkit worked well to trigger the interest and creativity of a very diverse group of participants. The youngest makers were 4 years old, the oldest about 60 . Some reported that they were professional hobbyist crafters, others were just curious about DIY technologies and digital fabrication. Overall, the activity seemed to create positive making experiences for people regardless of their different abilities, and no matter how short or long their attention span was. The feedback we received suggests that the flexibility in the activity allowed them to have fun and feelings of achievement:

- "It was fun - simple and rewarding and I liked being able to choose how I did it."

- "It was a lot of fun. I wasn't sure I'd be able to do it successfully but it was easy!"

- "I liked the combination of craftiness and technology."

\section{DISCUSSION}

Altogether the four use cases of FlexE-Touch illustrate the complex accumulation of real world influences on DIY electronics projects. They differ significantly in their respective motivations to employ capacitive touch sensing as well as in practical parameters such as cost, the availability of specialised tools, dedicated time, and location. While the instances of Tools for Wools and the Partnership Quilt resulted from collaborations of several crafters, the Light Writer emerged from the individual exploration of a new material. However, the craft collaborations also contrasted each other in that the participants were working locally (Partnership Quilt) or globally dispersed (Tools for Wools). Finally, the case of the public pop-up making even redefined the overall social constellation of contributors into the roles of those who participate and make, and those who facilitate the making. Setting up a stall at a local festival or community party meant that everyone passing by was invited to participate, and a stall host would be there to assist if needed.

In this discussion, we tease out some of the ways in which social and material complexities come together to make the use of a Schnittmuster meaningful and successful. We do this through a discussion of the ways in which the diversity of the audience, the material qualities and the importance of facilitation in the case study can be abstracted to the more theoretical framing of the Schnittmuster. After this, we also provide a critique of scalability, which has become of importance in HCI in recent years.

\section{Diversifying Maker Audiences}

Most noteworthy among all varieties across the use-cases is that we were able to engage such a diversity of people in hand-crafting capacitive touch sensor electrodes. We worked with people identifying as male or female, as young or old, as disabled or able-bodied, as locals, immigrants and/or from ethnic minorities, and as creative professionals or complete novices. Some who took part were more socially privileged than others, but others we worked with were girl scouts, sex workers, charity support workers, recipients of disability allowance, glass artists, quilting pattern designers, and knitting graffiti artists. FlexE-Touch helped us to leave settings formally framed around electronics and thereby reach out towards many different audiences besides the one already using maker spaces.

However, despite the very different project backgrounds, objectives, and social settings, all cases are technical instances of FlexE-Touch. At first, it might not seem very intuitive to use the same label for such differently looking design outcomes, but each of the cases employed the same Schnittmuster. FlexE-Touch defined the composition of craft material, electrode material, adhesive, connectors, and a signal-processing unit. It is a demonstratively simple and 'hacky' approach to technology, and its finesse and actual usefulness are based on its flexibility. A Schnittmuster enables the people using it to sensitively re-interpret and adapt the components to their contexts, requirements and constraints. As such, we regard it to be a valuable contribution for designers (especially those pursuing participatory, collaborative and exploratory approaches), and individuals who facilitate making activities for others (eg. teachers or organisers of maker spaces and workshops).

\section{Material Qualities}

FlexE-Touch focusses only on capacitive touch sensing. This is of course only one possible sensor input and ignores any other sensor and actuator types. We have not applied a Schnittmuster view on any other forms of sensors yet, but believe it is possible to do so with most basic electronics. As such, FlexE-Touch with its focus on capacitive touch sensing is a case study to theoretically and pragmatically explore the potentials of the concept. It is both source of (via our reflective practice recognizing that there were some meta-components being re-interpreted each time) and the first exemplar of a Schnittmuster. Future work will include applying the concept to other sensors, designing guidelines for developing new Schnittmuster, and providing more guidance on instantiating toolkits from a Schnittmuster.

We acknowledge that DIY electronics generally do not achieve the same technical precision as professionally fabricated components, however, we also appreciate that for learning and exploring new design potentials self-made 
electronics are often good enough. The motivation to make something that is not technically perfect but just 'good enough' can be very productive in the sense that it increases the accessibility of concepts that are perceived to be 'difficult to understand', and that it opens a space for creativity without being inhibited by the fear of making mistakes. For example, conductive paint has a higher resistance than metal and thus might not be the ideal choice for certain applications, however drawing might be less intimidating for electronics beginners than soldering.

Furthermore, this comes with a different quality for those designing or recreating DIY electronics. Doing electronic components yourself can feel more empowering than just combining ready-made blocks that keep secret how the sensor magic is happening [35]. The experience of crafting electronics using a technique that the novice maker already feels skilled or at least interested in, supports learning through personal meaning-making [9] and embeds the new skillset organically into previous knowledges. The professional quilters for example were not shy at all to work with such unusual materials such as aluminium foil, wires, and paper clips because it was still 'just' sewing.

A draw-back of creating DIY electronics that are just 'good enough' is that the crafted outcomes resulting from these novice experiments might be more suitable for prototypes rather than for final products based on their material qualities. For example, aluminum foil is cheap but it rips easily. The Tools for Wools prototype thus requires occasional bits of repairing after having been installed in different locations. However, we have also seen how the professional quilters in the Partnership Quilt project could significantly improve this method by strengthening and sewing the aluminum foil. Thus, there is a potential for learning from the toolkit users' expertise and for feeding this knowledge back into the range of possibilities how the Schnittmuster can be instantiated.

\section{Facilitation}

The Schnittmuster approach relies on the availability of pragmatic and empathic guidance for the novices. Considering the complexity surrounding different settings for making and the variety of needs, there is still a need for a facilitator role. Rather than a techno-solutionist idea that a single toolkit product can be universally usable regardless of context, we propose that every toolkit needs skilled 'tweaking' effort to make it work in a specific setting. However, a very specific skillset is required for the person taking on this role. To create a new Schnittmuster the facilitator needs both electronic expertise and some initial ideas how to recreate a component with alternative materials. Furthermore, a facilitator should be someone who is knowledgeable of the practical constraints, empathic to the toolkit users' requirements, and able to creatively and spontaneously adapt within the requisites of each specific use-case. This special profile combines technical and social skills to address the use-limiting issues of toolkits directly on the socio-technical level where they appear. However, this dependency on a highly skilled facilitator comes with new practical constraints. While material costs can be dramatically lowered, skilled work force is very expensive.

\section{Scalability}

Based on the limitations surrounding the material qualities and the importance of facilitation for novice makers, scalability, for example by packaging and commercialising it as a stand-alone product, is not the main focus for our Schnittmuster approach. However, developing and sharing Schnittmuster could be of particular value in contexts that favour providing equal opportunities to technology education over making profit. As we have seen in the FlexE-Touch use cases, a Schnittmuster can help novices regardless of their personal background to gain richer experiences and to create meaning around a provided kit.

We believe that the biggest advantage of Schnittmuster is that it can be used as an easily adaptable method to create DIY electronics outcomes that are 'good enough' for the particular project setting. Rather than setting out to design and deploy a finished 'omnipotent' toolkit product, it acknowledges that every project comes with different constraints and priorities. FlexE-Touch allowed us to tailor touch sensor toolkits 'good enough' for different settings and thereby to open up the activity of building sensor electrodes to a wider and more diverse audience. In this way, the Schnittmuster approach could be an interesting strategy for reaching more people and non-traditional contexts and to address issues of accessibility and technology democratisation around DIY-making practices.

\section{CONCLUSION}

In this paper, we presented a Schnittmuster approach to designing custom toolkits and illustrated it using the example of FlexE-Touch tailored for crafting capacitive touch sensor electrodes. A Schnittmuster is highly flexible and if used by empathic facilitators can react to a range of otherwise limiting project constraints. If funding is the matter, the costs can be lowered by using cheaper materials. If time and the availability of contributors are limited, it can adapt the manufacturing process by distributing the tasks. If learning about the underlying electronic concepts is the main goal, the activity can provide steps for guided recreation while maintaining space for personalisation and self-paced meaning-making. With the Schnittmuster, we contribute a novel critical perspective on and an alternative approach to designing maker toolkits that can assist facilitators of DIY electronics activities for novice makers.

\section{ACKNOWLEDGEMENTS}

This research was funded through the EPSRC CDT in Digital Civics (EP/L016176/1). Data supporting this publication is openly available under an 'Open Data Commons Open Database License'. Additional metadata are available at: http://dx.doi.org/10.17634/154300-61. Please contact Newcastle Research Data Service at rdm@ncl.ac.uk for access instructions. 


\section{REFERENCES}

1. Rafael Ballagas, Meredith Ringel, Maureen Stone, and Jan Borchers. 2003. iStuff: A Physical User Interface Toolkit for Ubiquitous Computing Environments. In Proceedings of the conference on Human factors in computing systems - CHI '03, 537. https://doi.org/10.1145/642611.642705

2. Jonathan Bean and Daniela Rosner. 2014. Making: Moevement or Brand? interactions 21, 1: 26-27. https://doi.org/10.1145/2541669

3. Viola van Beek. 2009. „Man lasse doch diese Dinge selber einmal sprechen". NTM Zeitschrift für Geschichte der Wissenschaften, Technik und Medizin 17, 4: 387-414. https://doi.org/10.1007/s00048-0090356-z

4. Leah Buechley and Hannah Perner-Wilson. 2012. Crafting Technology: Reimagining the Processes, Materials, and Cultures of Electronics. ACM Transactions on Computer-Human Interaction 19, 3: 1-21. https://doi.org/10.1145/2362364.2362369

5. Minjoo Cho and Daniel Saakes. 2017. Calm Automaton: A DIY Toolkit for Ambient Displays. In Proceedings of the 2017 CHI Conference Extended Abstracts on Human Factors in Computing Systems CHI EA '17, 393-396. https://doi.org/10.1145/3027063.3052968

6. David Cuartielles, Jonathan Bean, and Daniela Rosner. 2015. Conversations on Making. interactions 22, 1: 22-24. https://doi.org/10.1145/2685366

7. Lynn Dombrowski, Ellie Harmon, and Sarah Fox. 2016. Social Justice-Oriented Interaction Design: Outlining Key Design Strategies and Commitments. In Proceedings of the 2016 ACM Conference on Designing Interactive Systems - DIS '16, 656-671. https://doi.org/10.1145/2901790.2901861

8. Bruno Dumas, Denis Lalanne, Dominique Guinard, Reto Koenig, and Rolf Ingold. 2008. Strengths and weaknesses of software architectures for the rapid creation of tangible and multimodal interfaces. In Proceedings of the 2nd international conference on Tangible and embedded interaction - TEI '08, 47. https://doi.org/10.1145/1347390.1347403

9. Melanie Feinberg. 2017. Material Vision. In Proceedings of the 2017 ACM Conference on Computer Supported Cooperative Work and Social Computing - CSCW'17, 604-617. https://doi.org/10.1145/2998181.2998204

10. Nan-Wei Gong, Jürgen Steimle, Simon Olberding, Steve Hodges, Nicholas Edward Gillian, Yoshihiro Kawahara, and Joseph A. Paradiso. 2014. PrintSense: A Versatile Sensing Technique to Support Multimodal Flexible Surface Interaction. In Proceedings of the 32 d annual ACM conference on Human factors in computing systems - CHI '14, 1407-1410. https://doi.org/10.1145/2556288.2557173

11. Saul Greenberg and Chester Fitchett. 2001. Phidgets: Easy Development of Physical Interfaces through Physical Widgets. In Proceedings of the 14th annual ACM symposium on User interface software and technology - UIST '01, 209. https://doi.org/10.1145/502348.502388

12. Yasmin B. Kafai, Eunkyoung Lee, Kristin Searle, Deborah Fields, Eliot Kaplan, and Debora Lui. 2014. A Crafts-Oriented Approach to Computing in High School: Introducing Computational Concepts, Practices, and Perspectives with Electronic Textiles. ACM Transactions on Computing Education 14, 1, Article 1: 1-20. https://doi.org/10.1145/2576874

13. Yoshihiro Kawahara, Steve Hodges, Benjamin S Cook, Cheng Zhang, and Gregory D Abowd. 2013. Instant Inkjet Circuits: Lab-based Inkjet Printing to Support Rapid Prototyping of UbiComp Devices. In Proceedings of the 2013 ACM international joint conference on Pervasive and ubiquitous computing UbiComp '13, 363. https://doi.org/10.1145/2493432.2493486

14. Maker Faire. 2016. Media Center - Fast Facts. Retrieved September 4, 2017 from https://makerfaire.com/media-center/\#fast-facts

15. Janis Lena Meissner. 2015. Tools for Wools: Learning from and Designing for Urban Knitters. Master's Thesis. TU Wien (Vienna University of Technology). Retrieved from http://katalog.ub.tuwien.ac.at/AC12685309

16. Janis Lena Meissner. 2015. Tools for Wools: An Interactive Urban Knitting Installation and Creative Research Method. Proceedings of the 2015 ACM SIGCHI Conference on Creativity and Cognition: 337 338. https://doi.org/10.1145/2757226.2764769

17. Janis Lena Meissner and Geraldine Fitzpatrick. 2017. Urban Knitters on Interweaving Craft, Technologies and Urban Participation. In Proceedings of the 8th International Conference on Communities and Technologies - C\&T '17, 12-21. https://doi.org/10.1145/3083671.3083674

18. Janis Lena Meissner, John Vines, Janice McLaughlin, Thomas Nappey, Jekaterina Maksimova, and Peter Wright. 2017. Do-It-Yourself Empowerment as Experienced by Novice Makers with Disabilities. In Proceedings of the 2017 Conference on Designing Interactive Systems - DIS '17, 1053-1065. https://doi.org/10.1145/3064663.3064674

19. David A. Mellis, Sam Jacoby, Leah Buechley, Hannah Perner-Wilson, and Jie Qi. 2013. Microcontrollers as Material: Crafting Circuits with Paper, Conductive Ink, Electronic Components, and an "Untoolkit." In Proceedings of the 7th International Conference on 
Tangible, Embedded and Embodied Interaction - TEI '13, 83. https://doi.org/10.1145/2460625.2460638

20. David A Mellis and Leah Buechley. 2014. Do-ItYourself Cellphones: An Investigation into the Possibilities and Limits of High-Tech DIY. In Proceedings of the 32nd annual ACM conference on Human factors in computing systems - CHI '14, 17231732. https://doi.org/10.1145/2556288.2557309

21. Argyro Moraiti, Vero Vanden Abeele, Erwin Vanroye, and Luc Geurts. 2015. Empowering Occupational Therapists with a DIY-toolkit for Smart Soft Objects. In Proceedings of the Ninth International Conference on Tangible, Embedded, and Embodied Interaction TEI '14, 387-394. https://doi.org/10.1145/2677199.2680598

22. Simon Olberding, Nan-Wei Gong, John Tiab, Joseph a. Paradiso, and Jürgen Steimle. 2013. A cuttable multitouch sensor. In Proceedings of the 26th annual ACM symposium on User interface software and technology - UIST '13, 245-254. https://doi.org/10.1145/2501988.2502048

23. Simon Olberding, Michael Wessely, and Jürgen Steimle. 2014. PrintScreen: Fabricating Highly Customizable Thin-film Touch-Displays. In Proceedings of the 27th annual ACM symposium on User interface software and technology - UIST'14, 281-290. https://doi.org/10.1145/2642918.2647413

24. Geoff Payne. 2006. Social Divisions. Palgrave Macmillan, Basingstoke.

25. Hannah Perner-Wilson, Leah Buechley, and Mika Satomi. 2011. Handcrafting textile interfaces from a kit-of-no-parts. In Proceedings of the fifth international conference on Tangible, embedded, and embodied interaction - TEI' 11, 61 . https://doi.org/10.1145/1935701.1935715

26. Irene Posch. 2017. Crafting Tools. interactions 24, 2: 78-81. https://doi.org/10.1145/3038227

27. Irene Posch and Ebru Kurbak. 2016. CRAFTED LOGIC Towards Hand-Crafting a Computer. In Proceedings of the 2016 CHI Conference Extended Abstracts on Human Factors in Computing Systems CHI EA '16, 3881-3884. https://doi.org/10.1145/2851581.2891101

28. Mitchel Resnick. 1993. Behavior Construction Kits. Communications of the ACM 36, 7: 64-71. https://doi.org/10.1145/159544.159593

29. Yvonne Rogers, Jeni Paay, Margot Brereton, Kate L. Vaisutis, Gary Marsden, and Frank Vetere. 2014. Never Too Old: Engaging Retired People Inventing the Future with MaKey MaKey. In Proceedings of the 32 nd annual ACM conference on Human factors in computing systems - CHI '14, 3913-3922. https://doi.org/10.1145/2556288.2557184
30. Joel Sadler, Kevin Durfee, Lauren Shluzas, and Paulo Blikstein. 2015. Bloctopus: A Novice Modular Sensor System for Playful Prototyping. In Proceedings of the Ninth International Conference on Tangible,

Embedded, and Embodied Interaction - TEI '14, 347354. https://doi.org/10.1145/2677199.2680581

31. Jeffrey Sarmiento and Justin Marshall. 2017. Crafting Conductive Circuits in Glass. In Making Futures conference presentations, Plymouth College of Arts. Retrieved from http://makingfutures.plymouthart.ac.uk/acceptedabstracts/sarmiento-jeffrey/

32. Munehiko Sato, Ivan Poupyrev, and Chris Harrison. 2012. Touché: Enhancing Touch Interaction on Humans, Screens, Liquids, and Everyday Objects. $\mathrm{CHI}$ '12 Proceedings of the SIGCHI Conference on Human Factors in Computing Systems, c: 483-492. https://doi.org/10.1145/2207676.2207743

33. Martin Schmitz, Mohammadreza Khalilbeigi, Matthias Balwierz, Roman Lissermann, Max Mühlhäuser, and Jürgen Steimle. 2015. Capricate: A Fabrication Pipeline to Design and 3D Print Capacitive Touch Sensors for Interactive Objects. In Proceedings of the 28th Annual ACM Symposium on User Interface Software \& Technology - UIST '15, 253-258. https://doi.org/10.1145/2807442.2807503

34. Sowmya Somanath, Laura Morrison, Janette Hughes, Ehud Sharlin, and Mario Costa Sousa. 2016. Engaging "At-Risk" Students through Maker Culture Activities. In Proceedings of the TEI '16: Tenth International Conference on Tangible, Embedded, and Embodied Interaction - TEI '16, 150-158. https://doi.org/10.1145/2839462.2839482

35. Cristiano Storni. 2014. The problem of De-sign as conjuring: Empowerment-in-use and the politics of seams. In Proceedings of the 13th Participatory Design Conference on Research Papers - PDC '14, 161-170. https://doi.org/10.1145/2661435.2661436

36. Angelika Strohmayer and Janis Meissner. 2017. "We had tough times, but we've sort of sewn our way through it: the partnership quilt. XRDS: Crossroads, The ACM Magazine for Students 24, 2: 48-51. https://doi.org/10.1145/3155128

37. Nick Taylor, Ursula Hurley, and Philip Connolly. 2016. Making Community: The Wider Role of Makerspaces in Public Life. In Proceedings of the 2016 CHI Conference on Human Factors in Computing Systems - CHI '16, 1415-1425. https://doi.org/10.1145/2858036.2858073

38. Ron Wakkary, Markus Lorenz Schilling, Matthew a. Dalton, Sabrina Hauser, Audrey Desjardins, Xiao Zhang, and Henry W.J. Lin. 2015. Tutorial Authorship and Hybrid Designers. In Proceedings of the 33rd Annual ACM Conference on Human Factors in 
Computing Systems - CHI '15, 609-618. https://doi.org/10.1145/2702123.2702550

39. Maker Market Research | MAKE. Retrieved January 22, 2015 from http://makezine.com/hardwareinnovation-workshop/research/

40. Make Magazine: DIY Projects and Ideas for Makers. Retrieved September 7, 2017 from https://makezine.com/

41. Maker Faire. Retrieved September 7, 2017 from https://makerfaire.com/

42. Arduino. Retrieved September 4, 2017 from https://www.arduino.cc/

43. SparkFun Electronics. Retrieved September 4, 2017 from https://www.sparkfun.com/

44. Adafruit Industries. Retrieved September 4, 2017 from https://www.adafruit.com/

45. The Micro:bit Foundation. Retrieved September 4, 2017 from http://microbit.org/

46. Makey Makey. Retrieved September 4, 2017 from http://makeymakey.com/

47. littleBits: Award-winning electronic building blocks for creating inventions large and small. Retrieved September 11, 2017 from https://littlebits.cc/
48. Arduino Uno Rev3. Retrieved September 4, 2017 from https://store.arduino.cc/arduino-uno-rev3

49. LilyPad Arduino. Retrieved September 4, 2017 from https://www.arduino.cc/en/Main/ArduinoBoardLilyPad

50. Teensy USB Development Board. Retrieved September 4, 2017 from https://www.pjrc.com/teensy/

51. Touch Board - Bare Conductive. Retrieved September 11,2017 from https://www.bareconductive.com/shop/touch-board/

52. CapSense - Capacitive Sensing Library for Arduino. Retrieved September 12, 2017 from https:/playground.arduino.cc/Main/CapacitiveSensor?f rom=Main.CapSense

53. Adafruit MPR121 - 12-Key Capacitive Touch Sensor Breakout Tutorial | Adafruit Learning System. Retrieved September 12, 2017 from https://learn.adafruit.com/adafruit-mpr121-12-keycapacitive-touch-sensor-breakout-tutorial/overview 